Pacific Journal of Mathematics

EXTENSION OF CONTINUOUS FUNCTIONS ON 


\title{
EXTENSION OF CONTINUOUS FUNCTIONS ON TOPOLOGICAL SEMIGROUPS
}

\author{
Paul Milnes
}

\begin{abstract}
Examples show that functions of various kinds on subsemigroups of topological semigroups do not always extend to functions of the same kind on the containing semigroup. We show here that, if $S$ is a dense subsemigroup with identity of a topological group $G$, then there is a fairly large subspace of $C(S)$ whose members always extend at least to members of $C(G)$. As important applications of this theorem, we prove in this setting that the weakly almost periodic functions on $S$ extend to functions weakly almost periodic on $G$ and, in a somewhat more restricted setting, that the weakly almost periodic functions on $S$ are uniformly continuous. These results broaden the scope of answers we gave recently to some questions posed by $R$. Burckel. We also prove variants of some recent results of $A$. T . Lau and of $S$. J. Wiley, results concerning the extension of functions and the existence of invariant means on dense subsemigroups of topological groups.
\end{abstract}

1. Introduction. Suppose $S$ is a subsemigroup of a topological semigroup $T$. It follows directly from the definitions that the restriction to $S$ of a function continuous (left uniformly continuous) [weakly almost periodic] \{almost periodic\} on $T$ is continuous (left uniformly continuous) [weakly almost periodic] \{almost periodic\} on $S$. However, examples show that the converse is not true in general: a function of a specified kind on $S$ does not always extend to a function of the same kind on $T$. In this paper we show that, if $S$ is a dense subsemigroup with identity of a topological group $G$, then there is a subspace of $C(S)$ whose members always extend at least to members of $C(G)$. This subspace is fairly large - it always contains the left uniformly continuous functions - and was first introduced by $T$. Mitchell in his study [14] of the connection between fixed point properties that a topological semigroup $S$ might conceivably possess and the existence of different kinds of invariant means on subspaces of $C(S)$.

As applications of the extension theorem, which is itself a variant of a theorem of A. T. Lau [11], we prove variants of some recent results of S. J. Wiley [17]. More important applications are proofs that, if $S$ is a dense subsemigroup with identity of a topological group $G$, then every weakly almost periodic function on $S$ extends to a function weakly 
almost periodic on $G$, and that the weakly almost periodic functions on a dense subsemigroup with identity of any member of a broad class of topological groups are uniformly continuous. As well, we use our results to prove a variant of some results of Lau [11] concerning the existence of invariant means on dense subsemigroups of topologiclal groups.

The setting of some of the results proved in this paper is more general than that stipulated above.

2. Preliminaries. Let $S$ be a topological semigroup, that is, $S$ is a semigroup and a Hausdorff topological space, and the semigroup multiplication $(s, t) \rightarrow s t$ from the product topological space $S \times S$ into $S$ is continuous. We let $C(S)$ be the $C^{*}$-algebra of bounded continuous complex-valued functions on $S$ and denote by $\|f\|$ the supremum norm of $f \in C(S)$. Let $\beta S$ be the spectrum of $C(S)$, which is just the Stone-Čech compactification of $S$ if $S$ is completely regular. There is a canonical continuous map $e$ from $S$ into $\beta S: e(s) f=$ $f(s) \forall f \in C(S)$. Putting $e(S)=\{e(s) \mid s \in S\}$, we let $\sigma(C(S), e(S))$ denote the topology on $C(S)$ of pointwise convergence on $S$. If $f \in C(S)$ and $s \in S$, the left (right) translate $f_{s}\left(f^{s}\right)$ of $f$ by $s$ is defined by $f_{s}(t)=f(s t)\left(f^{s}(t)=f(t s)\right) \forall t \in S$. A subspace $X \subset C(S)$ is called translation invariant if $f_{s}, f^{s} \in X \forall f \in X$ and $\forall s \in S$.

Definition. The left $m$-introverted subspace of $C(S)$ is defined to be $\left\{f \in C(S) \mid\right.$ the function $s \rightarrow x\left(f_{s}\right)$ is a member of $C(S)$ for each $x \in \beta S\}$. We call this subspace $L M C(S)$.

The subspace $L M C(S)$ was introduced by Mitchell [14] and the notation is his. It follows immediately from definitions that $L M C(S)$ is a translation invariant $C^{*}$-subalgebra of $C(S)$, that, if $S_{1}$ is a subsemigroup of a topological semigroup $S$, then the restriction to $S_{1}$ of members of $L M C(S)$ are in $L M C\left(S_{1}\right)$ (i.e., $\left.\left.L M C(S)\right|_{s_{1}} \subset L M C\left(S_{1}\right)\right)$, and that $w^{*}(L M C(T)) \subset L M C(S)$ whenever $S$ and $T$ are topological semigroups and $w$ is a continuous homomorphism of $S$ into $T$. We proved in [12] that $L M C(S)$ can be characterized as $L M C(S)=$ $\left\{f \in C(S) \mid\left\{f^{s} \mid s \in S\right\}\right.$ is relatively $\sigma(C(S), e(S))$-compact $\}$.

Let $\mu S$ denote the spectrum of $L M C(S)$ and let $m$ be the canonical continuous map from $S$ into $\mu S$. In [12] we showed that multiplication can be canonically defined in $\mu S$ so that $\mu S$ becomes a compact semigroup for which the map $x \rightarrow \dot{x} y$ from $\mu S$ into $\mu S$ is continuous $\forall y \in \mu(S)$ and the map $x \rightarrow y x$ is continuous at least for $y \in m(S)=$ $\{m(s) \mid s \in S\}$, though usually not for every $y \in S$. We also proved that $\mu S$ with this multiplication has a universal mapping property: if $v$ is a continuous homomorphism from $S$ onto a dense subset of a compact semigroup $T$ in which the maps $x \rightarrow v(s) x, s \in S$, and $x \rightarrow x y, y \in T$, are 
all continuous from $T$ into $T$, then $\exists$ a unique continuous homomorphism $w$ from $\mu S$ onto $T$ such that $(w(m)(s))=v(s) \forall s \in S$.

The left uniformly continuous subspace $L U C(S)$ of $C(S)$ consists of those members $f$ of $C(S)$ for which $\left\|f_{s_{\alpha}}-f_{s}\right\| \rightarrow 0$ whenever $s_{\alpha} \rightarrow s$ all in $S$. The right uniformly continuous subspace $R U C(S)$ is defined analogously using right translates and the uniformly continuous subspace $U C(S)$ equals $L U C(S) \cap R U C(S)$. The inclusions $L U C(S) \subset$ $L M C(S) \subset C(S)$ follow directly from the definitions, all three spaces coinciding if $S$ is discrete or compact.

Two more subspaces of $C(S)$ will be needed later, the weakly almost periodic subspace $W A P(S)$ and the almost periodic subspace $A P(S)$. By definition $W A P(S)=\left\{f \in C(S) \mid\left\{f_{s} \mid s \in S\right\}\right.$ is weakly relatively compact in $C(S)\}$ and $A P(S)=\left\{f \in C(S) \mid\left\{f_{s} \mid s \in S\right\}\right.$ is relatively compact in $C(S)\} . A P(S) \subset L U C(S)$ and $A P(S) \subset W A P(S) \subset$ $L M C(S)$ in general, all four spaces being equal to $C(S)$ if $S$ is compact. Also, $W A P(S)(A P(S))$ is a translation invariant $C^{*}$ subalgebra of $C(S)$ in whose spectrum $\omega S(\alpha S)$ multiplication can be defined in a canonical way so that $\omega S(\alpha S)$ becomes a compact semigroup with separately continuous multiplication (a compact topological semigroup) with the following universal mapping property [15; (6.2) Theorem]: if $w(a)$ is the canonical continuous map of $S$ into $\omega S(\alpha S)$ and $v$ is a continuous homomorphism of $S$ onto a dense subset of a compact semigroup with separately continuous multiplication (of a compact topological semigroup) $T$, then there is a unique continuous homomorphism $u$ of $\omega S(\alpha S)$ onto $T$ such that $u(w(s))=v(s)$ $(u(a(s))=v(s)) \forall s \in S$. General references for matters concerning $W A P(S)$ and $A P(S)$ are $[1,3]$.

3. The extension theorem. We now prove the extension theorem mentioned in the introduction; this theorem, which is a variant of a theorem of Lau [11; Theorem 2.3], was proved in a less general setting, that of dense subgroups of topological groups, in [12]. The proof here is adapted from that in [12] and is given for completeness.

THEOREM 1. Let $S$ be a dense subsemigroup with identity of a topological semigroup $G$ that is algebraically a group. (We do not assume $G$ has continuous inversion.) Then every $f \in L M C(S)$ extends to a function in $C(G)$, i.e., $\left.C(G)\right|_{s} \supseteq L M C(S)$.

Proof. We produce a contradiction from the assumption that $\exists s \in G$ and nets $\left\{s_{\alpha}\right\}$ and $\left\{t_{\beta}\right\}$ in $S$ such that $\lim _{\alpha} s_{\alpha}=s=\lim _{\beta} t_{\beta}$ and $a=\lim _{\alpha} f\left(s_{\alpha}\right) \neq \lim _{\beta} f\left(t_{\beta}\right)=b$. Without loss, we may assume that $\lim _{\alpha} e\left(s_{\alpha}\right)=x \in \beta S$; thus $x(f)=a$. Since $S$ is dense in $G$, there is a net $\left\{r_{\gamma}\right\} \subset S$ such that $\lim _{\gamma} r_{\gamma}=s^{-1} \in G$. Now consider the values of $f$ along 
the "triple net" $\left\{t_{\beta} r_{\gamma} s_{\alpha}\right\}$. Since $f \in L M C(S)$, the function $s \rightarrow x\left(f_{s}\right)$ is continuous on $S$ and, since $t_{\beta} r_{y} \rightarrow e$, the identity of $G$ and of $S$, by continuity of multiplication, $x\left(f_{t_{\beta_{\gamma}}}\right)$ should approach $x\left(f_{e}\right)=x(f)=a$. But $x\left(f_{t_{\beta} r_{\gamma}}\right)=\lim _{\alpha} f\left(t_{\beta} r_{\gamma} s_{\alpha}\right)$ is close to $f\left(t_{\beta}\right)$ for all large enough $\gamma$ since $f \in C(S)$ and $r_{\gamma} s_{\alpha} \rightarrow e$. This implies that $x\left(f_{t_{\beta r_{\gamma}}}\right) \rightarrow \lim _{\beta} f\left(t_{\beta}\right)=b \neq a$ as $t_{\beta} r_{\gamma} \rightarrow e$, the desired contradiction.

The following examples, both due to T. Mitchell, show what can happen if $S$ is not required to be dense in $G$, or if $G$ is not required to be a group.

EXAMPLE 1 [6; p. 257]. Consider the open semi-infinite interval $(0, \infty)$, a subsemigroup of the usual additive real numbers $R$. The function $t \rightarrow \sin (1 / t)$ defined on $(0, \infty)$ is left uniformly continuous there and hence is in $\operatorname{LMC}((0, \infty))$, but has no continuous extension to the group $R$ (or, for that matter, to the subsemigroup $[0, \infty)$ of $R$ in which $(0, \infty)$ is dense).

EXAMPLE 2 [14]. Let $S_{0}=S \cup\{0\}$ be the one-point compactification of the free semigroup $S$ on two generators, with multiplication extended from $S$ to $S_{0}$ by $0 \cdot s=s \cdot 0=0 \cdot 0=0 \forall s \in S$. Then $S_{0}$ is a compact topological semigroup, $S$ is dense in $S_{0}$ and $L M C(S)=C(S)$, but the only functions in $C(S)$ that extend to functions in $C\left(S_{0}\right)$ are those that have a limit at infinity.

Recently Wiley [17] proved the following results:

(i) If $S$ is an abelian subsemigroup of a compact topological group $G$, then every $f \in L U C(S)$ extends to a member of $L U C(G)$, i.e., $\left.L U C(G)\right|_{s}=\operatorname{LUC}(S)$.

(ii) If $S$ is an abelian subsemigroup of a topological group $G$ and $S$ has compact closure, then again $\left.L U C(G)\right|_{s}=L U C(S)$. These results are proved using the theorem that a compact cancellation semigroup is a group [8; Theorem 9.16] and a theorem of Katětov [10] which asserts that every bounded function that is uniformly continuous on a subspace of a uniform space extends to a bounded function uniformly continuous on the containing space. With Wiley's methods and the fact that

$$
C(G)=L M C(G)=L U C(G)=W A P(G)=A P(G)
$$

for compact groups $G$, we can get the following corollaries to Theorem 1. We note as well that the equality $L U C(S)=A P(S)$ holds in Wiley's setting.

COROLlaRy 2. If $S$ is a subsemigroup with identity of a compact topological group $G$, then every $f \in L M C(S)$ extends to a function in $L M C(G)$, i.e., $\left.\operatorname{LMC}(G)\right|_{s}=\operatorname{LMC}(S)$. Hence $L M C(S)=A P(S)$. 
COROllary 3. If $S$ is a dense subsemigroup with identity of a topological group $G$ and $S$ has compact closure in $G$, then $\left.L U C(G)\right|_{S}=$ $\operatorname{LMC}(S)$.

One is prompted to ask: In the settings under consideration, does it ever happen that $\left.A P(G)\right|_{s}=A P(S)$ or, if this fails, at least $\left.W A P(G)\right|_{s} \supset A P(S)$ ?

It is clear that $\left.A P(G)\right|_{S}=A P(S)$ in the settings where $G$ is compact. And in [13] it is shown that, if $H$ is any subgroup containing more than two elements of the group $G$ of finite permutations of the natural numbers, then $\left.A P(G)\right|_{H} \not \supset A P(H)$. However, for noncompact $G$ positive results are available in some settings. These results are consequences of Theorem 1 above, Wiley's result, some theorems of Berglund [2; Proposition 4 and Corollary 11] and Theorem 2 of [13], which asserts that each function weakly almost periodic on an open subgroup of a locally compact group extends to a function weakly almost periodic on the containing group.

THEOREM 4. If $S$ is a subsemigroup with identity of a locally compact abelian group $G$ and the closure of $S$ in $G$ is a group, then every $f \in A P(S)$ extends to a member of $A P(G)$, i.e., $\left.A P(G)\right|_{s}=$ $A P(S)$. The conclusion still holds if $S$ does not contain the identiy and the closure of $S$ is required to be merely compact.

THEOREM 5. If $S$ is a subsemigroup with identity of a locally compact group $G$ and the closure of $S$ in $G$ is an open subgroup of $G$, then every $f \in W A P(S)$ extends to a member of $W A P(G)$, i.e., $\left.W A P(G)\right|_{s}=W A P(S)$.

REMARK. In [12] an example is given which shows that the conclusion of Theorem 4 (Theorem 5) can fail if $G$ is not required to be abelian (if the closure of $S$ is not required to be open). See the remark following Theorem 7 ahead.

The next theorem, which we need in section 4 , is a sharpening of Theorem 2 of Rao [16] which deals with topological groups complete in an invariant metric. The proof is an adaptation of Rao's proof.

THEOREM 6. Let $G$ be algebraically a group and topologically a complete metric space. Suppose the maps $s \rightarrow$ st and $s \rightarrow$ ts from $G$ onto $G$ are continuous for all $t \in G^{1}$. Then $L U C(G)=L M C(G)$.

' It has been pointed out to us that the hypotheses here imply that $G$ is a topological semigroup. (See Theorem 1 in D. Montgomery's, Continuity in topological groups, Bull. Amer. Math. Soc., 42 (1936), 879-882.) 
Proof. We assume $G$ is not discrete and must prove $L M C(G) \subset$ $L U C(G)$. Suppose $f \in C(G), f \notin L U C(G)$. We show $f \notin L M C(G)$.

If $f \notin L U C(G), \exists \epsilon>0, s^{\prime} \in G$ and sequences $\left\{s_{n}^{\prime}\right\},\left\{t_{n}^{\prime}\right\} \subset G$ such that $s_{n}^{\prime} \rightarrow s^{\prime}$ and $\left|f\left(s_{n}^{\prime} t_{n}^{\prime}\right)-f\left(s^{\prime} t_{n}^{\prime}\right)\right| \geqq \epsilon \forall n$. We write $t_{n}=s_{n}^{\prime} t_{n}^{\prime}, s_{n}=s^{\prime} t_{n}^{\prime}$ and note that $t_{n} s_{n}^{-1} \rightarrow e$ as $n \rightarrow \infty$. By taking subsequences if necessary and forming a linear combination of $f$ and the constant function 1 , we may assume $\lim _{n} f\left(s_{n}\right)=1, \lim _{n} f\left(t_{n}\right)=0$. Consider now the sequence $\left\{f^{s_{n}}\right\}$ of right translates of $f$. At least one of the following two situations must arise.

1. $\exists$ a sequence $\left\{r_{k}\right\} \subset G, r_{k} \rightarrow e$, and a subsequence $\left\{f^{s_{m}}\right\}$ of $\left\{f^{s_{n}}\right\}$ satisfying $f^{s_{m}}\left(r_{k}\right) \rightarrow p_{k}$ as $m \rightarrow \infty$ with $\left|p_{k}\right| \leqq \frac{1}{2}$ for each $k$. If $x$ is a cluster point in $\beta G$ of $\left\{e\left(s_{m}\right)\right\}$, then $\left|x\left(f_{r_{k}}\right)\right|=\lim _{m}\left|f^{s_{m}}\left(r_{k}\right)\right|=\left|p_{k}\right| \leqq \frac{1}{2} \forall k$, while $\quad x\left(f_{e}\right)=x(f)=\lim _{m} f\left(s_{m}\right)=1$. Since $r_{k} \rightarrow e$, this implies $f \notin L M C(G)$.

2. If $d$ denotes the metric in $G$, there is a ball $D(e, \delta)=$ $\{r \in G \mid d(e, r)<\delta\}$ with $\delta>0$ and a subsequence $\left\{f^{s_{m}}\right\}$ of $\left\{f^{s_{n}}\right\}$ such that $\left|f^{s_{m}}(r)\right| \leqq \frac{1}{2}$ for only finitely many $m$ for each $r \in D(e, \delta)$. We let $y$ be a cluster point in $\beta G$ of $\left\{e\left(t_{m}\right)\right\}$, hence $y(f)=0$, and find a sequence $\left\{v_{k}\right\}_{k=1}^{\infty} \subset G$ such that $v_{k} \rightarrow e$ and $\left|y\left(f_{v k}\right)\right| \geqq \frac{1}{2}$. This will imply $f \notin L M C(G)$.

For a fixed $k$, we find $v_{k}$ as follows. Choose $w_{k} \in G$ such that $w_{k} \neq e$ and $d\left(w_{k}, e\right)<2^{-k} \delta$, let $D_{k}=\overline{D\left(w_{k}, d\left(w_{k}, e\right) / 2\right)}$ (closure in $\left.G\right)$, and let $\quad E_{m}=\left\{r \in D_{k}|| f^{s_{m}}(r) \mid \geqq \frac{1}{2}\right\}$. Since $\quad D_{k} \subset D(e, \delta), \quad D_{k}=$ $\cup_{i \leqq 1}\left(\cap_{m>i} E_{m}\right)$. The Baire category theorem [4; p. 20] applied to $D_{k}$ asserts that $\exists i_{0}$ such that $\cap_{m>i_{0}} E_{m}$ has nonvoid interior in $D_{k}, E^{0}$, say. Thus $\exists v_{k} \in E^{0}$ and $b_{k}>0$ such that $D\left(v_{k}, b_{k}\right) \subset E^{0}$. Explicity, $\forall r \in D\left(v_{k}, b_{k}\right)$ and $\forall m>i_{0},\left|f^{s_{m}}(r)\right| \geqq \frac{1}{2}$. If we now observe that, for a given $\epsilon>0,\left|y\left(f_{v_{k}}\right)-e\left(t_{m}\right) f_{v_{k}}\right|<\epsilon$ for infinitely many $m$, that $v_{k} t_{m} s_{m}^{-1} \rightarrow v_{k}$ as $m \rightarrow \infty$ and hence that $\left|e\left(t_{m}\right) f_{v_{k}}\right|=\mid f^{s_{m}}\left(v_{k} t_{m} s_{m}^{-1} \mid \geqq \frac{1}{2} \forall\right.$ large $m$, we see that $\left|y\left(f_{v_{k}}\right)\right| \geqq \frac{1}{2}$ and we are done.

REMARKS. (i) Mitchell [14] proved that $L M C(G)=L U C(G)$ for locally compact topological groups $G$.

(ii) One might consider a variant of Theorem 2 with "complete metric" replaced by "locally compact". A powerful theorem of Ellis [5] could then be applied which asserts that the group $G$ is a topological group and reduces this case to that dealt with by Mitchell.

(iii) In [12] we gave examples of semigroups $S$ for which $L M C(S)$ is not equal to $L U C(S)$; however, these are not topological semigroups, the multiplication being only continuous in each variable separately.

The preceding theorems, examples and remarks lead one to the Questions. Is there a topological group $G$ for which $\operatorname{LMC}(G) \neq L U C(G)$ ? Or is there a subsemigroup $S$ of a topological 
group for which $\operatorname{LMC}(S) \neq \operatorname{LUC}(S)$ ? With $S$ and $G$ as in Theorem 1, can it happen that $\left.L M C(G)\right|_{s} \neq L M C(S)$ ?

\section{Applications to weakly almost periodic} functions. The following results are proved in R. Burckel's monograph [3; pp. 42, 44]:

(a) If $G$ is a locally compact topological group, then every function in $W A P(G)$ is uniformly continuous.

(b) Let $G$ be a commutative topological group, $S$ a dense subgroup. Then $W A P(S)=\left.W A P(G)\right|_{s}$.

Burckel asked [3; p. 81] if the local compactness hypothesis is necessary in (a) and if the commutativity hypothesis is necessary in (b). Among the results proved in [12] were answers to these questions. In Theorems 7 and 8 we now widen the scope of these answers. We believe Theorem 8 presents the first broad class of semigroups that are not discrete and not groups and for which it is known that the weakly almost periodic functions are uniformly continuous.

THEOREM 7. If $S$ is a dense subsemigroup with identity of a topological semigroup $G$ that is algebraically a group, then every function in $W A P(S)(A P(S))$ extends to a function in $W A P(G)$ $(A P(G))$, i.e., $\left.W A P(G)\right|_{s}=W A P(S)\left(\left.A P(G)\right|_{s}=A P(S)\right)$.

REMARK. In [12] we gave an example to show how badly the conclusion of Theorem 3 can fail if $S$ is not required to be dense in $G$. For the example, $G$ is the affine group of the line (the " $a x+b$ group"), a locally compact topological group, and $S$ is the closed normal abelian subgroup of $G$ that is isomorphic to the additive reals. It was shown that any extension to $G$ of a nontrivial character on $S$ cannot be uniformly continuous and hence is not weakly almost periodic ${ }^{2}$.

THEOREM 8. Let $S$ be a dense subsemigroup with identity of a semigroup $G$ that is a locally compact topological group or that is a topological semigroup which is algebraically a group and topologically a complete metric space. Then each function in WAP $(S)$ is uniformly continuous.

Proof of Theorem 7. We carry out the proof for $W A P(S)$. The proof for $A P(S)$ is similar.

${ }^{2}$ C. Chou, in Weakly almost periodic functions with zero mean, Bull. Amer. Math. Soc. 80 (1974), 297-299, has announced an example where this behaviour occurs and $G / S$ is compact. 
Since $W A P(S) \subset L M C(S)$, we know that each $f \in W A P(S)$ extends to a function in $C(G)$. The proof can be completed by referring to a theorem of Berglund [2; Proposition 4] or by using the universal mapping property of the spectrum of $W A P(G)$ that was mentioned at the end of $\S 2$. We follow the latter course. By Theorem 1, WAP $(S)$ is (canonically isomorphic to) a $C^{*}$-subalgebra of $C(G)$. Hence, if $e$ is the canonical continuous map of $G$ into $\beta G$ and, $\forall s \in G, v(s)$ is the restriction of $e(s)$ to $W A P(S)$, the map $s \rightarrow v(s)$ is a continuous homomorphism of $G$ onto a dense subset of $\omega S$. It follows from the universal mapping property of $\omega G$ that $v$ factors canonically through $\omega G$ : there is a continuous homomorphism of $\omega G$ onto $\omega S$ mapping the canonical image of $s$ in $\omega G$ onto $v(s) \in \omega S \forall s \in G$. This implies that $W A P(S)$ is (canonically isomorphic to) a $C^{*}$-subalgebra of $W A P(G)$. The proof is complete.

Proof of Theorem 8. The hypotheses ensure that $L M C(G)=$ $L U C(G)$ (Theorem 6 and Remark (i) following) and that each function in $W A P(S)$ extends to a function in $W A P(G)$ (Theorem 7). Since $W A P(G) \subset L U C(G)$ and the restriction to $S$ of a function uniformly continuous on $G$ is uniformly continuous, we are done.

5. Another application. For $S$ a dense subsemigroup of a topological group $G$, Lau [11] proved that:

(i) If $C(G)$ has a left invariant mean (LIM), then $U C(S)$ has a LIM.

(ii) If $L U C(G)$ has a LIM and $S$ has the finite intersection property for right ideals, then $U C(S)$ has a LIM.

(iii) If $S$ has the finite intersection property for right ideals and the finite intersection property for left ideals, and $U C(G)$ has a LIM, then $U C(S)$ has a LIM.

The next theorem, an easy consequence of Theorem 1, is a variant of these results of Lau, which were also proved by extension of functions, and almost, but not quite, subsumes the result (i). We omit the proof.

THEOREM 9. If $S$ is a dense subsemigroup with identity of a topological semigroup $G$ that is algebraically a group, and if $C(G)$ has a LIM, then LMC $(S)$ has a LIM. Hence, if $S$ is a dense subsemigroup with identity of a compact group, LMC $(S)$ has a (unique) LIM.

An As example to which our Theorem 9 applies and to which Lau's (ii) and (iii) above do not apply is as follows.

EXAMPLE. As is well known, the 3-dimensional rotation group $G$ contains a free group $G_{0}$ on two generators. Let $a, b \in G$ generate $G_{0}$ 
and let $S$ be the topological subsemigroup of $G$ generated by $a, b$ and the identity. Then $a S$ and $b S$ are right ideals of $S a S \cap b S=$ $\varnothing$. Since $a S \cap b S=\varnothing$. Since the closure of $S$ in $G$ is a compact group [8; Theorem 9.16] it follows from our results that $L M C(S)$ admits a (unique) LIM.

Example 2 following Theorem 1 shows that conclusion conslusion of Theorem 9 can fail to hold if $G$ is not required to be a group. $C\left(S_{0}\right)$ there has a LIM, namely evaluation at 0 , while $C(S)$ does not have a LIM.

To show that the conclusion of Theorem 9 can fail if $S$ is not required to be dense in $G$, we have an example of quite a different kind, one for which $G$ is discrete, and hence, the reason for the failure of the theorem is not that $\left.C(G)\right|_{s}$ does not contain much of $C(S)$. A "generic" example of this type was given by Hochster [9].

EXAmpLe. Let $G$ be the affine group of the line, which can be represented as $G=\{(a, b) \mid a, b \in R, a>0\} \quad$ with $(a, b)$ $(x, y)=(a x, a y+b)$. We $(a, b)(x, y)=(a x, a y+b)$. We show that $G$ contains a free semigroup on two generators. Let $c$ be a real number and consider the subsemigroup $S$ generated by $A=(c, 0), B=(1,1)$ and the identity $e=(1,0)$. Any member $C \neq e$ of $S$ can be written in the form

$$
C=A^{n_{1}} B^{m_{1}} A^{m_{2}} B^{m_{2}} \cdots A^{n_{1}} B^{m_{1}} A^{n_{1+1}}
$$

where $m_{1}, m_{2}, \cdots, m_{i}, n_{2}, n_{3}, \cdots, n_{i} \geqq 1, n_{1}, n_{i+1} \geqq 0$. Putting $\sum_{j=1}^{k} n_{j}=N_{k}$, $k=1,2, \cdots, i+1$, we see that $C=\left(c^{N_{i+1}}, \sum_{k=1}^{i} m_{k} c^{N_{k}}\right)$. If we choose $c$ transcendental, then the correspondence given by $*$ between members of $S \backslash\{e\}$ and finite sequences of integers $\left(n_{1}, m_{1}, n_{2}, m_{2}, \cdots, n_{i}, m_{i}, n_{i+1}\right)$ with $m_{1}, m_{2}, \cdots, m_{i}, n_{2}, n_{3}, \cdots, n_{i} \geqq 1, n_{1}, n_{i+1} \geqq 0$ is one-to-one, i.e., $S \backslash\{e\}$ is free. $S$ is not discretely contained in $G$ in that $\exists$ sequences $\left\{A_{n}\right\},\left\{B_{n}\right\} \subset S$ such that $A_{n} B_{n}^{-1} \rightarrow(1,0)$ and $B_{n}^{-1} A_{n} \rightarrow(1,0)$ in the metric topology. So we consider $G$ as a discrete group. As such, $C(G)=$ $L U C(G)=U C(G)$ has a LIM, since $G$ is a semidirect product of abelian groups [7; Theorems 1.2.1 and 2.3.3], while $C(S)=L U C(S)=$ $U C(S)$ does not.

\section{REFERENCES}

1. J. F. Berglund and K. H. Hofmann, Compact Semitopological Semigroups and Weakly Almost Periodic Functions, Lecture Notes in Mathematics, 42, Springer-Verlag, Berlin, 1967.

2. J. F. Berglund, On extending almost periodic functions, Pacific J. Math., 33 (1970), 281-289.

3. R. B. Burckel, Weakly Almost Periodic Functions on Semi-groups, Gordon and Breach, New York, 1970. 
4. N. Dunford and J. T. Schwartz, Linear Operators I, Interscience, second printing, New York, 1964.

5. R. Ellis, Locally compact transformation groups, Duke Math. J., 24 (1957), 195-202.

6. E. Granirer and A. T. Lau, Invariant means on locally compact groups, Illinois J. Math., 15 (1971), 249-257.

7. F. P. Greenleaf, Invariant Means on Topological Groups and Their Applications, D. Van Nostrand, Princeton, 1969.

8. E. Hewitt and K. Ross, Abstract Harmonic Analysis I, Springer-Verlag, Berlin, 1963.

9. M. Hochster, Subsemigroups of amenable groups, Proc. Amer. Math. Soc., 21 (1969), 363-364.

10. M. Katětov, On real-valued functions in topological spaces, Fund. Math., 38 (1951), 85-91, and correction, Fund. Math., 40 (1953), 203-205.

11. A. T. Lau, Invariant means on dense subsemigroups of topological groups, Canad. J. Math., 23 (1971), 797-801.

12. P. Milnes, Compactifications of semitopological semigroups, J. Australian Math. Soc., 15 (1973), 488-503.

13. - On the extension of continuous and almost periodic functions, (to appear in Pacific J. Math.).

14. T. Mitchell, Topological semigroups and fixed points, Illinois J. Math., 14 (1970), 630-641.

15. J. S. Pym, The convolution of functionals on spaces of bounded functions, Proc. London Math. Soc., 15 (1965), 84-104.

16. C. R. Rao, Invariant means on spaces of continuous or measurable functions, Trans. Amer. Math. Soc., 114 (1965), 187-196.

17. S. J. Wiley, Extensions of left uniformly continuous functions on a topological semigroup, Proc. Amer. Math. Soc., 33 (1972), 572-575.

Received March 23, 1974. This research was supported in part by NRC grant A7857.

The University of Western Ontario, London, Canada 


\section{PACIFIC JOURNAL OF MATHEMATICS}

\section{EDITORS}

RICHARD ARENS (Managing Editor)

University of California

Los Angeles, California 90024

\section{J. DugundJI}

Department of Mathematics University of Southern California Los Angeles, California 90007

D. Gilbarg and J. Milgram

Stanford University

Stanford, California 94305

\section{ASSOCIATE EDITORS}
E. F. BECKENBACH
B. H. NeumanN
F. WoLF
K. YoshiDA

\section{SUPPORTING INSTITUTIONS}

\author{
UNIVERSITY OF BRITISH COLUMBIA \\ CALIFORNIA INSTITUTE OF TECHNOLOGY \\ UNIVERSITY OF CALIFORNIA \\ MONTANA STATE UNIVERSITY \\ UNIVERSITY OF NEVADA \\ NEW MEXICO STATE UNIVERSITY \\ OREGON STATE UNIVERSITY \\ UNIVERSITY OF OREGON \\ OSAKA UNIVERSITY
}

\author{
UNIVERSITY OF SOUTHERN CALIFORNIA \\ STANFORD UNIVERSITY \\ UNIVERSITY OF TOKYO \\ UNIVERSITY OF UTAH \\ WASHINGTON STATE UNIVERSITY \\ UNIVERSITY OF WASHINGTON \\ AMERICAN MATHEMATICAL SOCIETY
}

The Supporting Institutions listed above contribute to the cost of publication of this Journal, but they are not owners or publishers and have no responsibility for its contents or policies.

Mathematical papers intended for publication in the Pacific Journal of Mathematics should be in typed form or offset-reproduced (not dittoed), double spaced with large margins. Underline Greek letters in red, German in green, and script in blue. The first paragraph or two must be capable of being used separately as a synopsis of the entire paper. Items of the bibliography should not be cited there unless absolutely necessary, in which case they must be identified by author and Journal, rather than by item number. Manuscripts, in duplicate, may be sent to any one of the four editors. Please classify according to the scheme of Math. Reviews, Index to Vol. 39. All other communications should be addressed to the managing editor, or Elaine Barth, University of California, Los Angeles, California, 90024.

100 reprints are provided free for each article, only if page charges have been substantially paid. Additional copies may be obtained at cost in multiples of 50 .

The Pacific Journal of Mathematics is issued monthly as of January 1966. Regular subscription rate: $\$ 72.00$ a year (6 Vols., 12 issues). Special rate: $\$ 36.00$ a year to individual members of supporting institutions.

Subscriptions, orders for back numbers, and changes of address should be sent to Pacific Journal of Mathematics, 103 Highland Boulevard, Berkeley, California, 94708.

PUBLISHED BY PACIFIC JOURNAL OF MATHEMATICS, A NON-PROFIT CORPORATION Printed at Jerusalem Academic Press, POB 2390, Jerusalem, Israel.

$$
\begin{gathered}
\text { Copyright (C) } 1975 \text { Pacific Journal of Mathematics } \\
\text { All Rights Reserved }
\end{gathered}
$$




\section{Pacific Journal of Mathematics}

\section{Vol. 58, No. $2 \quad$ April, 1975}

Zvi Artstein and John Allen Burns, Integration of compact set-valued functions . . . . . . . . . 297

Mark Benard, Characters and Schur indices of the unitary reflection group $[321]^{3} \ldots \ldots \ldots . .309$

Simeon M. Berman, A new characterization of characteristic functions of absolutely continuous

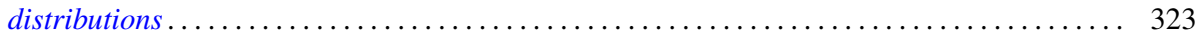

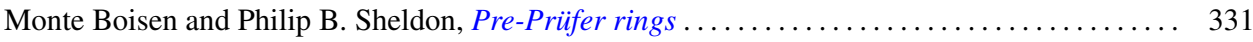

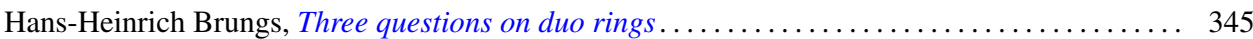

Iracema M. Bund, Birnbaum-Orlicz spaces of functions on groups................. 351

John D. Elwin and Donald R. Short, Branched immersions between 2-manifolds of higher

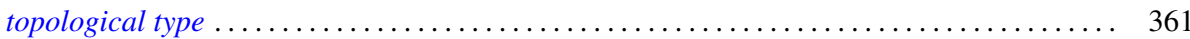

Eric Friedlander, Extension functions for rank 2, torsion free abelian groups . .......... 371

Jon Froemke and Robert Willis Quackenbush, The spectrum of an equational class of

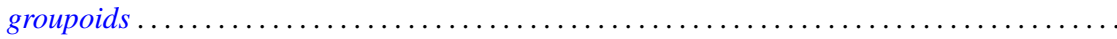

Barry J. Gardner, Radicals of supplementary semilattice sums of associative rings ...........

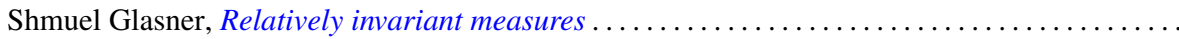

George Rudolph Gordh, Jr. and Sibe Mardesic, Characterizing local connectedness in inverse

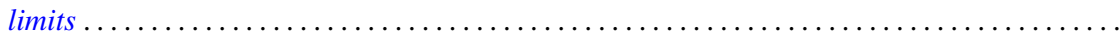

Siegfried Graf, On the existence of strong liftings in second countable topological spaces......

Stanley P. Gudder and D. Strawther, Orthogonally additive and orthogonally increasing

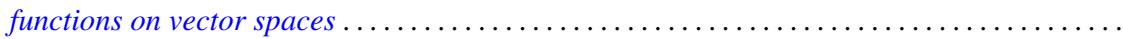

Darald Joe Hartfiel and Carlton James Maxson, A characterization of the maximal monoids and

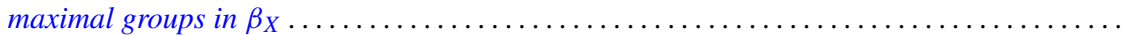

Robert E. Hartwig and S. Brent Morris, The universal flip matrix and the generalized faro-shuffle. .

William Emery Haver, Mappings between ANRs that are fine homotopy equivalences. .

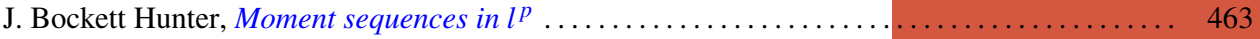

Barbara Jeffcott and William Thomas Spears, Semimodularity in the completion of a poset.... 467

Jerry Alan Johnson, A note on Banach spaces of Lipschitz functions . . . . . . . . . . . . 475

David W. Jonah and Bertram Manuel Schreiber, Transitive affine transformations on

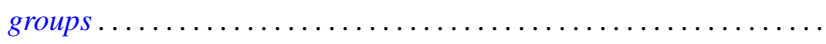

Karsten Juul, Some three-point subset properties connected with Menger's characterization of

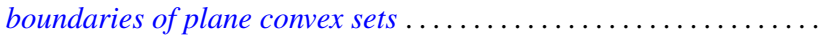

Ronald Brian Kirk, The Haar integral via non-standard analysis . . . . . . . . . . . . . 517

Justin Thomas Lloyd and William Smiley, On the group of permutations with countable

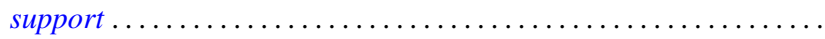

Erwin Lutwak, Dual mixed volumes .................................. 531

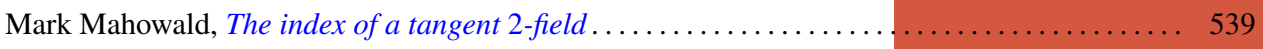

Keith Miller, Logarithmic convexity results for holomorphic semigroups . . . . . . . . . . . . 549

Paul Milnes, Extension of continuous functions on topological semigroups . . . . . . . . . . 553

Kenneth Clayton Pietz, Cauchy transforms and characteristic functions ................ 563

James Ted Rogers Jr., Whitney continua in the hyperspace $C(X) \ldots \ldots \ldots \ldots \ldots \ldots \ldots \ldots .569$

Jean-Marie G. Rolin, The inverse of a continuous additive functional . . . . . . . . . . . . 585

William Henry Ruckle, Absolutely divergent series and isomorphism of subspaces . ........ 605

Rolf Schneider, A measure of convexity for compact sets . ..................... 617

Alan Henry Schoenfeld, Continous measure-preserving maps onto Peano spaces .......... 627

V. Merriline Smith, Strongly superficial elements .......................... 643

Roger P. Ware, A note on quadratic forms over Pythagorean fields . . . . . . . . . . . . . . 651

Roger Allen Wiegand and Sylvia Wiegand, Finitely generated modules over Bezout rings . . . . 655

Martin Ziegler, A counterexample in the theory of definable automorphisms . . . . . . . . . 665 